\title{
Measuring the mass of Kepler-78b using nonparametric Gaussian process estimation
}

\author{
Samuel K. Grunblatt ${ }^{1}$, Andrew W. Howard ${ }^{1}$ \\ and Raphaëlle D. Haywood ${ }^{2}$ \\ ${ }^{1}$ Institute for Astronomy, University of Hawaii, \\ 2680 Woodlawn Drive, Honolulu, HI 96822 \\ email: skg@ifa.hawaii.edu \\ ${ }^{2}$ SUPA School of Physics and Astronomy, University of St Andrews, \\ North Haugh, St Andrews, Fife KY16 9SS, United Kingdom
}

\begin{abstract}
Measuring the masses of rocky planets is quite difficult, as the relevant signal produced by such planets is often dwarfed by stellar activity by an order of magnitude or more. Developing a more robust way to isolate the stellar activity in these measurements is crucial to the search for Earth-like planets. We estimate the mass of Earth-size planet Kepler-78b using a Gaussian process estimator to describe the stellar activity in both photometric and radial velocity (RV) data, confirming previous results with a more robust technique that can be extended toward Earth analogues.
\end{abstract}

Keywords. eta-Earth, Gaussian process, radial velocity, etc.

Kepler-78b is a transiting planet with a radius of $1.16 \pm 0.16 \mathrm{R}_{\oplus}$ that orbits a young, active $\mathrm{K}$ dwarf in 8 hours (Sanchis-Ojeda et al. (2013)). The mass of Kepler-78b was reported from RV measurements using the HIRES and HARPS-N spectrographs. Even in quiet stars, stellar RV variations are an order of magnitude larger than the RV signal of Earth, and this effect is magnified in young stars (Hillenbrand et al. (2015)). Howard et al. (2013) and Pepe et al. (2013) independently modeled the RV signal of Kepler-78 parametrically to remove stellar activity while measuring the planetary signal.

In this study, we model the RVs using a quasiperiodic GP estimator trained on Kepler photometry. Using the package emcee, we determine parameter distributions with a Monte Carlo Markov Chain to simultaneously model the quasiperiodic stellar activity and Keplerian signal, allowing us to measure a mass of $1.87 \pm 0.27 \mathrm{M}_{\oplus}$.

We find that despite the stellar activity producing radial velocity shifts ten times the size of the planetary signal, we can robustly isolate the planetary signal. This technique is dependent on using the more frequently sampled photometric signal to constrain the stellar rotation period, thereby restricting the Gaussian process estimator to a period distinct from the planetary orbital period. This technique is most valuable for obtaining parameters for small, rocky planets or planets at large orbital distances with orbital periods distinct from the stellar rotation. For more details, see Grunblatt et al. (2015).

\section{References}

Grunblatt, S., Howard, A., \& Haywood, R. 2015 ApJ, 808,127

Hillenbrand, L., Isaacson, H., Marcy, G. et al. 2014, Cool Stars, 18, 759

Howard, A., et al. 2013, Nature, 503, 381

Pepe, F., et al. 2013, Nature, 503, 377

Sanchis-Ojeda, R., Rappaport, S., Winn, J., et al. 2013, ApJ, 774, 54 